\title{
Breeding for Resistance to Husk Spot Disease in Macadamia ${ }^{\dagger}$
}

\author{
Jasmine Nunn 1,*, Mobashwer Alam 1, Olufemi Akinsanmi ${ }^{2}$, Craig Hardner ${ }^{2}$ and Bruce Topp ${ }^{1}$ \\ 1 QAAFI, University of Queensland, Nambour, QLD 4560, Australia; m.alam@uq.edu.au (M.A.); \\ b.topp@uq.edu.au (B.T.) \\ 2 QAAFI, University of Queensland, Brisbane, QLD 4067, Australia; o.akinsanmi@uq.edu.au (O.A.); \\ c.hardner@uq.edu.au (C.H.) \\ * Correspondence: j.nunn@uq.edu.au \\ † Presented at the third International Tropical Agriculture Conference (TROPAG 2019), Brisbane, Australia, \\ 11-13 November 2019.
}

Published: 13 February 2020

\begin{abstract}
In Australian macadamia orchards, significant decreases in saleable nuts have been reported due to husk spot caused by the fungal pathogen, Pseudocercospora macadamiae. In some cultivars, husk spot induces early fruit abscission. This may result in under developed nuts and consequently, increased proportions of commercial rejects. While fungicides are able to reduce impacts of husk spot, breeding for resistance offers a cost-effective and sustainable control option. Quantification of genetic variation for resistance in Australian macadamia breeding germplasm will assist selection. Previous husk spot studies have utilised different inoculation methods including the insertion of diseased husk into tree canopies and the direct application of $P$. macadamiae suspensions to fruit. Insertion of diseased husk best reflects natural infection processes; however, it requires rainfall or overhead irrigation to disperse spores and may result in the delivery of unequal quantities of inoculum. Different disease parameters have also been measured to compare susceptibility, including proportions of diseased fruit, mean number of lesions per fruit or mean diameter of chlorosis or necrotic lesions. It is unknown which of these parameters are most valuable to assess when screening for resistance. Determining the proportions of variation in such phenotypic traits that is due to genetic variance could identify which of these will better respond to selection. Finally, the use of marker assisted selection may reduce time and costs of disease screening, but no studies have investigated associations between markers and susceptibility to husk spot. In this study, breeding progeny and parents and wild accessions will be screened for husk spot resistance. Repeatability of different inoculation methods will be compared. The heritability of parameters used to measure husk spot incidence and severity will be estimated. Analysis will be undertaken to identify genetic markers associated with resistance. Screening of a wide genetic base for husk spot resistance will enable selection of parents for current breeding. Optimisation of inoculation and phenotypic screening methods and the identification of markers associated with resistance will increase the efficiency of future trials.
\end{abstract}

Keywords: husk spot; Pseudocercospora; macadamia; disease resistance; plant breeding

Funding: This research was funded by Hort Innovation, using the macadamia research and development levy and contributions from the Australian Government. Hort Innovation is the grower-owned, not-for-profit research and development corporation for Australian horticulture. The author acknowledges the Research Training Program Stipend. 
Conflicts of Interest: The author declares no conflict of interest.

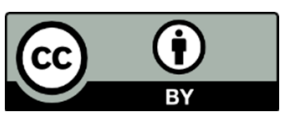

(C) 2020 by the authors. Licensee MDPI, Basel, Switzerland. This article is an open access article distributed under the terms and conditions of the Creative Commons Attribution (CC BY) license (http://creativecommons.org/licenses/by/4.0/). 